\title{
A New Model of the Birth of the Universe
}

\author{
Alexey Belyaev \\ Independent Researcher, Russia ${ }^{\mathrm{i}}$
}

Copyright $(2017$ by authors, all rights reserved. Authors agree that this article remains permanently open access under the terms of the Creative Commons Attribution License 4.0 International License

\begin{abstract}
This paper studies the principles laid in existing models and the approaches to building new models and shown that the existing models of the birth of the Universe not only require an introduction of a new category "dark energy" to explain the accelerated dispersion of matter but that they do not fulfill Hubble's law, which was a necessary before the discovery of the accelerated expansion of the Universe. It is found a new cosmological model could be built and the principles are studied with which the new model of the birth of the Universe must comply.
\end{abstract}

Keywords Dark Energy, Hubble's Law, Accelerated Expansion, Birth of the Universe, Big Bang Theory

\section{Introduction}

Modern views on the possible models of the birth and evolution of the Universe are very clearly and sequentially presented in $[1,2]$. In the Big Bang theory, and in its updated and extended version - the inflationary model, it is suggested that a cloud of matter appears in empty space, in the inflationary model - in vacuum, with equivalent in all directions velocities of its elements that are chaotically scattered in space and that make up the parent cloud of the Universe. The methods of converting energy into matter are described in the framework of cosmology with a certain degree of reliability.

It is a strong assumption that the Universe has a time of birth. Theoretically, let's take an option, not of a true birth of the Universe with the formation of a substance, but of cyclic changes in the eternal Universe density and types of contracting and expanding matter after its explosion from its compacted state. In this situation, the substance exists eternally but undergoes qualitative changes during the cyclic life of the Universe. However, this case brings up so many questions that it becomes difficult to always adhere to the hypothesis of an eternally existing substance (but hypothetically possible).

Potentially, the expansion of the Universe in compliance with Hubble's law which simultaneously fulfills the principle of homogeneity may be considered in the case of an infinite
Universe that does not have a single moment of birth and can exist for an infinitely long time. However, this implies that, even though no discussion is made about the accelerated expansion of the Universe, the dark energy must exist. In addition, there is an explicit difficulty in the understanding of the term "infinity". Thus, homogeneity of the Universe in this case, should be understood that there is no specific location in space, that the expansion of the Universe when viewed from another galaxy is identical to the observations of an observer on Earth. An everlasting inhomogeneous Universe is also possible. But it becomes quite difficult to imagine and speculate when combining the theory with the observational facts.

The existence of an isolated point in time at which the Universe began its existence follows from Friedman's solutions, as he was trying to mathematically describe the space-time world. In 1922 - 1924 he created a non-stationary model of the Universe, laying the foundations of the theory of the expanding Universe. In 1929 Hubble discovered the dynamics of celestial bodies associated with the expansion of matter in the Universe, which gave reason for the creation of the Big Bang theory which describes the birth of the Universe. The fact of the matter is that the Friedman model and the Hubble distribution may be interconnected if the birth of the Universe is considered as an explosive process that occurs under certain conditions. An ordinary explosion model implies that the initial high pressure at the heart of the explosion leads to a scattering of debris with large radial velocities quickly decreases leading to a sharp decrease in radial velocities of the debris that follow. But what can be considered pressure or debris in the Big Bang? In 1946 Gamow proposed to tie the Big Bang model to the model of a "hot universe" by predicting the possibility of the existence of electromagnetic cosmic background radiation and its expected temperature. In 1964 Penzias and Wilson discovered the existence of the cosmic microwave background radiation, which led to the final acceptance of the Big Bang model combined with the model of the hot Universe.

In the Friedman model matter (substance and radiation) is considered as a continuous medium (as a liquid) evenly distributed in the space and having at any arbitrary time specific values of mass density and pressure. The Friedman 
solution lies in formulating equations of energy, motion and continuity in the four-dimensional space-time, that take into account the resistance of the gravitational forces to the expanding in the space of the cloud, which is made up of a "continuous medium". The desire to expand is due to the internal pressure in the cloud (though the term "pressure" can exist solely due to electromagnetic interference, it is somehow absent from the model, the surface tension forces are also absent, there is only the pressure of itself). Thereby, macro characteristics of our Universe (open, flat or closed) are definitely dependent on the mass of the Universe.

It is important to note that the solutions in Friedman model may only be considered for a specific short time interval, because all the constants in the equations are time-dependent, i.e., changing in the process of evolution of the Universe. Moreover, there are other nuances that are discussed in this paper, that hinder the application of Friedman model.

The accelerated dispersion of matter in the Universe was discovered at the turn of the last century, which not only violated Hubble's law, but also necessitated a complete abandonment of the Friedman model. However, the model of the birth of the Universe, which was based on the principles of Friedman's model, had already been recognized, so it was necessary to continue using it: the basic assumptions become part of the consciousness as unquestionable. Therefore, as a solution to this hopeless situation an unprecedented step was made - an introduction into physics of a new category of an unknown nature "dark energy", which solved the problem.

Today it is assumed that the Universe emerged from a single point of concentrated energy in space, which, after a short period of time in a certain stage of evolution of the Universe, turned into homogeneous plasma cloud consisting mostly of protons and electrons. The observed cosmic microwave background radiation today is associated with the released radiation as a result of the recombination of plasma. The homogeneity of the released radiation, if it is considered a relic, can only be explained by high spatial uniformity of plasma at the time of its recombination. The formation of galaxies and stars had started, according to modern views, after the recombination of plasma in a homogenous plasma cloud from which the Universe is composed.

Thus, the model the birth of the Universe adopted today is conceptually based on the appearance of a very homogeneous cloud of matter at a certain stage of evolution, which obeys the laws of statistics and is categorized by thermodynamic performance. Scientists have many unanswered questions related to the way this step of evolution is to be achieved. In this paper, however, the emphasis is not on how to form a uniform cloud of matter in the Universe, but on the very fact of its appearance, which is now considered to be indispensable.

\section{Aims}

The introduction of an unknown dark energy into physics allowed us to keep the adopted model of the birth of the Universe, which is based on the principles of Friedman's model. Furthermore, the Friedman model as a whole can be combined with Hubble's law. But the question of how well this can be done has move to the background with the introduction of the dark energy and has almost ceased to exist at all. Consequently, the first aim of this paper is to discuss the weight of the existing shortcomings of combining the Friedman model with Hubble's law.

Additionally, new opinions on the cosmological processes have recently surfaced in the press that make Friedman model completely unsuitable for cosmology (see $[3,4]$ ). Therefore, the second aim is to concisely present information on these new publications in relation to the justification for using the existing model of the birth of the Universe.

But the main aim of this work is to determine whether the accumulated knowledge would be enough to in principle to create a new model of the birth of the Universe, which is based on the available experimental data.

\section{The Friedman Model and the Hubble Law}

If we look at the relationship between the Friedman model and the Hubble law from a descriptive point of view, i.e., in terms of visual and logical perception, which helps us form mental images, and serves as the only mechanism of awareness, and not using mathematical logic, which only helps us control the compliance of reality with the mathematical models without becoming aware of the possible ways to implement the embedded mathematical principles into existing physical environment, the following picture will appear.

The uniform cloud of material can be divided into equal micro-volumes and the number of their constituent molecules can be connected with each micro-volume, i.e., divide it into micro-portions with equal masses. In the process of expansion of the cloud only its outer boundary moves, thus, increasing the volume of the cloud, and the entire inner zone, which is fixed by the time of the observation of the border displacement, remain immobile. But the centers of mass of the micro-portions of the substance, nevertheless, will start to move from the center of mass of the homogeneous cloud in radial directions under the influence of internal pressure because the growth of the volume of the cloud will take place no because of the emergence of new micro-portions of the substance, but due to the growth in volume of the existing micro-portions. The main assumption of this model is the instant alignment of pressure of the expanding cloud throughout its volume. Under this condition, which cannot be met in reality, Hubble's law will be fulfilled: the further away from the selected micro-portion of the substances is any other micro-portion, the greater the rate of divergence of its center of mass from the point of observation.

Friedman's mathematical model, which studies the expansion of uniform cloud, is based on the assumption that 
the expanding cloud can instantly equalize the pressure to fit the entire volume. It is for this reason that the Friedman's model, considering the uniform cloud, can be combined with Hubble's law. It is obvious that with time the rate of expansion of the cloud, with decreasing internal pressure, decreases. This means that the proportionality coefficient between the velocity of the center of mass of the micro-portions and the distance from it to the observation point arbitrarily selected within the cloud, i.e. Hubble constant, will decrease. In doing so, the Hubble distribution itself under the condition of instantaneous pressure equalization will continue to be supported; only the constant will decrease.

If the opposing forces (internal pressure and gravity) suddenly disappear, and the selected micro-portions transform into independent objects, then the Hubble constant for diverging objects created will become fixed. But if only the pressure is disappears and the forces of gravity remain, then diverging objects will, firstly, decelerate and, secondly, do so unevenly. This means that the Hubble distribution law must be violated.

The process of independent contraction of the various "micro-portions" of the substance, i.e., processes of creation of space objects, disrupt cloud uniformity and eliminate the pressure existing in it. Taking into account the assumption that the estimated age of the Universe is 13.7 billion years, and that plasma recombination occurred about 12.7 billion years ago, it is obvious that Hubble law is not compatible with the adopted model of the birth of the Universe. Thus, however surprising it might sound, the Hubble law does not confirm the accepted model of the Big Bang, but rather contradicts it. It is useful to note that at the time of formation of the Big Bang model the discussion about dark energy had not taken place.

In this case, the physical principles embedded in the general structure of the model of the birth and development of the Universe with the transition from the stage of a homogeneous expanding cloud to the stage of the disappearance of pressure in an already heterogeneous cloud are discussed. These stages should be described using different mathematical apparatus. But the general conceptual approach, without a mathematical description, shows that there is no correct model of the birth of the Universe in terms of the physical terminology.

\section{The Problems of the Big Bang Model and Lambda-CDM Model}

The Big Bang Model has seriously aided our understanding of the world order. However, the problem mentioned above is not the only problem of this model.

The main problem of the model of the Big Bang is the presence of a singular point of infinite mass density and temperature from which the Universe began its development. It is not that the density of the material and the temperature cannot be simultaneously infinitely large, but in the basic inability to observe a singular point by an external observer, for who time had stopped in the neighborhood of the point. The fact is physics can be used to describe the observed phenomenon in practice. In 1967 , the need to recognize the existence of a singular point had been confirmed mathematically by Hawking in his analysis of the process of expansion of the Universe in the framework of general relativity. However, the laws of physics are powerless to describe the physical nature of this point, it is a product of logic and mathematics, but it cannot be considered in physical terms. It is important to note that the identified problem is so serious that the existence of any other hypothetical possibility of describing the birth of the Universe, even only conceptually relying on available observational facts with a lot of reservations would have immediately rejected the existing model.

In reverse chronological history of existence and evolution of our Universe it was determined that Planck length cannot be achieved (our Universe does not roll into a single point). That is why the inflationary model appeared, specifying the standard of the Big Bang model.

In 1981 the theory of cosmological inflation emerged and has since been considerably strengthened and elaborated upon, timidly and uncertainly pushed the question of the existence of a singular point a little into the background. The inflation model initially introduced space-time, which began to expand exponentially at a rate which soon exceeded the speed of light, but the substance began forming only after $10^{-35}$ seconds, presumably in local oscillations of the stretched zone and not in a single point. However, no clearly selected types of variants of the emerging substance and the occupied matter generating space appeared for these first moments of the birth of the Universe. Moreover, it is important to highlight that the notion of a singular point is still present in the description of black holes, which have been woven into the theory of cosmological inflation.

The Big Bang model and the inflationary model are the same in their basic principles. A creation of a homogeneous plasma cloud of the Universe takes place in both models. However, the inflationary model as the more progressive one, has become strengthened by new hypotheses about dark energy, dark matter, black holes, and has become dominant.

In spite of the progress made by the inflationary model, it generates many new uncertainties and new factors: it is not certain what the physical essence of the initial scalar field is, the physical fullness of the initial quantum fluctuations, the evolution of vacuum, the ongoing processes and the spatial zone of matter generation, the principle of inertia in relation to inflation, which did not stop but only changed its speed and others. According to the inflationary model, the main stage of the expansion of space-time, the stage of "inflation", took place before the substance came to existence. However, after the substance was created the expansion of space-time did not stop, but only entered a new restrained stage. What theorists understand by the criterion of "Hubble constant" in 
relation to the space-time, including the period in which the matter did not exist, and how this criterion may be associated with the movement of the created matter, they cannot explain themselves. Simply no explanations exist. But most importantly, if we hypothetically assume that the observer appeared before the birth of the Universe, then, in the absence of substance, it would be impossible to understand what is time and what is space. The presence of substance in nothingness or in vacuum gives meaning to these categories. Even if we consider the expansion of space-time for the period when the substance was already in existence, uncertainties still remain, especially in interconnectedness of space-time and matter. Will this change the law of inertia in the continuously expanding space-time; will the physical properties of the substance change; how can a metric of something affect something physical, if the metric is not considered as an ether, etc. ? - the list of unanswered questions extensive. So, to say that it is not the material that expands in space, occupying all large spatial areas, but the space-time is the same as not saying anything. Along the way, it is necessary to note that the expanding space-time could not have cooled the relic electromagnetic radiation, which did not yet exist at the time of intensive expansion of space-time. Moreover, if the expanding space-time does reduce the kinetic energy of the photons, then in any case, it does so without complying with Wien's displacement law in Planck's spectrum.

As a result of the preliminary analysis of the status quo, we can say that today there is no plausible model of birth of the Universe, which can describe the available experimental observations.

\section{Published Data Indicating the Non-applicability of the Currently Accepted Model of the Birth of the Universe}

In article [3] it is argued that the free photon gas, i.e., photons, which are not in equilibrium with the substance and do not interact with it, do not have the ability to interact with each other and form a thermodynamic system that can heat or cool. This is so obvious that it does not require additional comments which, nevertheless, are made in this paper. However, due to historical circumstances, the same thermodynamic laws were applied to the photon gas, which allegedly escaped from captivity after the plasma recombination, as for the related photon gas. As a result, the discovered cosmic microwave background radiation was wrongly identified as a relic. It is a mistake if only for the simple reason that the plasma recombination process is not instantaneous, but gradual (respectively, the relic background radiation cannot have the Planck spectrum for a particular equilibrium temperature), or non-colliding photons must have long left that zone, which was occupied by the expanded plasma, which was once in equilibrium with radiation (the relict photons cannot be observed from earth even if cosmological inflation is taken into account, especially because at the time when the relict background radiation was recognized no assumptions about the expansion of space-time had been made). If we supposed this assumption was wrong, it would lead to a need for a different explanation of the origin of the cosmic radiation, other than being plasma residue after recombination. The conclusion made in [3] that the background radiation cannot be a relic, is so obvious, that the error in recognizing the choice of its source is only a matter of time.

Article [4] shows that in the framework of classical gravity, the accelerated dispersion of matter in the Universe is a natural consequence of the contraction of the cloud composed of elements that are able to combine with each other in collisions and unite their masses. This shows that, firstly, there is no need for dark energy and, secondly, that the movement of galaxies, for which the impact of pressure of the expanding uniform cloud ceases, have no connection to the Friedman model. In other words, the existence of homogeneous plasma in the early stages of evolution of the Universe is not a necessary and sufficient criterion. The discussion in [4] is so simple and clear that their recognition is also only a matter of time.

But the conclusion made in [3,4] is no less important. Both articles independently and from different starting positions came to the same conclusion that the Universe is surrounded by a massive and, on average, uniform halo. This conclusion is very significant not only for a better understanding of the evolution of the Universe and the causes of unsuitability of the existing models, but also for the creation of new cosmological models of the Universe of birth.

Firstly, the halo of the Universe allows, without any partial reticence and reservations, to understand the origin of both the cosmic microwave background radiation and the cosmic $\mathrm{X}$-ray background radiation.

Secondly, expanding in the empty space the cloud is unable to form dense outer layers. Therefore, the current ideological picture of the existence of a cloud in the early stages of the evolution of the Universe is incorrect.

Thirdly, a very important factor is that a self-evident and necessary to explain the modern structure of the Universe fact of the necessity of existence of a parent cloud of matter can still be correct if something is added to it that will give the elements of the cloud, as explained in [4], primary component of velocity in radial directions toward the periphery. Then the possibility of halo becomes feasible and self-evident. This conclusion, which draws attention to the need for the elements of the original cloud of matter to have preferential radial velocities, leads to the conclusion that the recognition of the halo around the Universe additionally entails a heuristic load in the search process for new variants of models of creation of the Universe. 


\section{Analysis of the Current Situation. A Bit about Vacuum}

The above analysis shows that the existing models of the birth of the Universe need to be replaced, and that to date, no model exists, which can be used to complement it with the new position of the existence of a halo of the Universe.

Before trying to outline the approaches that are required to create a new model of the birth of the Universe, we have to decide on where the Universe was born: in empty space or in a vacuum. This is the fundamental question for future models.

The theory of cosmological inflation, which is predominant today, is based on the concept of "vacuum". Vacuum is the basis of quantum field theory. Therefore, its existence is widely recognized today. But there is still no understanding of what the vacuum actually is. Most scientists cannot even say whether it exists as a substance or as a concept.

Nobody yet knows what vacuum is. It's energy distributed in empty space. The type of energy that an unobserved scalar field can generate that fills all the infinite space is clouded with mystery. What's more the basic definition of energy in physics is mechanistic, however, vacuum existed before the appearance of substance (in the inflationary theory vacuum existed even before space-time). The existence of vacuum is generally accepted but there are no tips and no assumptions that can help us understand it. It is completely neutral, as if it is not there at all. Regardless of the actual methods of the implementation of the electromagnetic and gravitational interactions, understanding their nature, the vacuum does not prevent the physical manifestations of the forces of these fields, does not conduct electromagnetic waves (is not an ether, which conducts electromagnetic waves) and allows space-time, in the case of its existence, to realize its curvature. Vacuum does not interfere with the mechanical movements, does not affect the stability of elementary particles or anything formed from them. On the other hand, the elementary particles are formed in emptiness from energy only due to the existence of vacuum. In fact, all quantum effects exist only because vacuum exists.

In modern understanding, vacuum is a self-existing quantum field that does not consider, even a hypothetical possibility, the existence of distant sources of this field. It is a basic state of a quantum field with minimum energy and quantum numbers with a zero value, but which has the ability to create a "vacuum condensate", which is a non-zero value of the "vacuum average". What the "vacuum condensate" and "vacuum average" are can only be explained mathematically, no physical interpretation is possible. We'll have to introduce the concepts of "Hilbert space", "Fock space", "degrees of freedom", etc. However, we can say that vacuum condensate correspond to the fluctuating fields with zero-point oscillations, generating virtual particles. But it is fundamentally impossible to explain what energy is, in relation to a vacuum and what the virtual particles, "vacuum degeneracy" and "false vacuum" are in physical terms. It is impossible to guess or imagine, using the modern approach to its description, how vacuum may be represented as a physical object. After all, a quantum field that completely fills everything does not even have a source. In the modern sense - it's just a materialized mathematical environment.

The uncertainties in the understanding of the physical nature of vacuum leads to the fact that the hypothesis of the possible origin of the Universe is connected, not only with the problem of the spatial region of its birth including the question of the singularity, but also with the problem of the theoretically possible processes that accompanied the birth. Modern scientific knowledge of the foundation of the world around us is still very modest. Therefore, the discussions about the birth of the Universe, at this stage, can only be schematic and abstract, with a discussion of the trends, but without the decryption of specific physical mechanisms of their implementation.

We know, from modern physics, that the birth of particle pairs in an absolute emptiness comes with the mandatory supply of energy to the place of birth with the indispensable presence of vacuum. If a pair of virtual "particle antiparticle", existing within the vacuum theory, of a time shorter than the time-uncertainty relation for the energy of the particles, are given energy, the pair of virtual particles can turn into a pair of real particles. In this case, the field is able to transfer energy to the nascent pair "particle antiparticle" as well. Therefore, pairs of "particle antiparticle" can be spontaneously born in severely inhomogeneous electric or gravitational fields.

Modern framework of physics assumes that no pairs "particle - antiparticle" can be born without vacuum. But at the same time the particles produced in vacuum cannot get anything from it, otherwise we would have to accept vacuum as an ether. The vacuum in the particle pair production processes acts only as a catalyst. It exchanges energy, but, in the end retains its original state. As a result, the pair "particle - antiparticle" is born in an emptiness and is made up of energy.

The observed phenomenon of the birth of an electron-positron pair with the disappearance of gamma-quantum in the vicinity of an atomic nucleus today is interpreted as gamma rays colliding with high-energy virtual photon generated by spatially severely inhomogeneous electric field, and their energy is converted into the energy of the produced particle pairs. A virtual photon in this observation is required: an electron-positron pair has zero total momentum, so it cannot be formed from just a single photon carrying the momentum; the law of conservation of momentum requires the involvement of a second photon. There is no clear relationship between vacuum and quantum electromagnetic field. But the mathematical apparatus for all types of quantum fields is the same. Therefore separately coexisting vacuum and the quantum electromagnetic field theories are identical in their construction, but develop independently, without establishing the relationship between 
them. The topic of whether vacuum in quantum electrodynamics is a form of all filling void that exists in the absence of an electromagnetic field, is not raised. However, vacuum is seen in the state where the principal quantum number of oscillators is equal to zero. The Feynman diagrams are intended to give a clear physical interpretation of the processes involving quantum fields.

The above example of electron-positron pair refers to its appearance in largely non-uniform electric field with the disappearance of the gamma-quantum. But the birth of the pair "particle - antiparticle", spontaneous or otherwise, is possible in severely inhomogeneous gravitational fields. However, the likelihood of the spontaneous formation of particles with non-zero rest mass is small even near massive black holes. Taking into account not only virtual "particle antiparticle" pairs, but also virtual photons are born in vacuum, Hawking showed, in 1974, that black holes generate electromagnetic radiation with the spectrum of a black body heated to a certain temperature, that depends only on the black hole's mass. In this paper we do not discuss the problems associated with such calculations, evaporation of the black holes has not yet been proven experimentally, but only demonstrate that in modern interpretations vacuum plays an important role in the process of production of "particle - antiparticle" pair.

The possibility of spontaneous pair production in severely non-uniform fields is clearly and readily explained by the work done by the external field to break the connections of electrically oppositely charged virtual elementary particles. Practically, this means that the direction of deviation of the particles is not equally probable but is defined by the external field. At the same time, it should not be forgotten that the produced particle and antiparticle fly away from the place of birth along a common straight line. No other processes of substance formation from vacuum are currently known to science. Thus, firstly, vacuum exists and is active and, secondly, the situation where the direction divergence of the newborn particles in vacuum are established beforehand, has a right to exist.

We know that the birth of the Universe is connected with some kind of action, which allowed a huge number of elementary particles to be created with the complete absence of antiparticles in the immediate vicinity. There is no consideration, even hypothetical, in modern physics that only particles, without antiparticles, can be produced from energy in a void in a presence of vacuum. Such assumptions are contrary to modern knowledge. However, if we consider possible historical variants of spatial accumulation of antimatter in the Universe, then the ideology of emergence in the early stages of the birth of the Universe of a uniform cloud has led to overlooking the very possibility of the acceptability of such a hypothesis. Possible variants of appearance of asymmetry between matter and antimatter in the models of expanding and cooling cloud of the Universe have begun to be developed. The fact of the matter is that the theory of a singular point does not allow a simultaneous generation of multiples of "particle - antiparticle" pairs. The sequence of the first moments of the life of the Universe is as follows: first, quark-gluon plasma is born from a homogeneous and isotropic area with abnormally high energy; it then gives life to the equally probable spatial directions baryogenesis processes, accompanied by the emergence and subsequent annihilation of antimatter. The main problem is to develop an asymmetric theory in such a way, that it does not to eliminate all of the substance in the processes of annihilation with the complete elimination of all the antimatter. This is a very difficult problem.

Thus it is considered today that the birth of the Universe was a momentary phenomenon and the possibility that an avalanche like growth of the acts of creation of the "particle antiparticle" pairs at the moment of birth of the Universe is not even hypothetically assumed. However, there is no direct evidence that the matter making up the Universe was created in one moment. Nothing prevents the source of the substance from working from the moment of the birth of the Universe to the present day. Although, if that were really the case, then there would be clues indicating the existence of a continuously running generator. As it is, there have been no observations pointing to this version of the evolution of the Universe. However, nothing prevents us from developing models of the birth of the Universe that take into account the dampened oscillations in the ongoing acts of generation of the "particle-antiparticle" pairs. In other words, the process of creation of the Universe could well have lasted for a finite period of time within a finite volume of space with numerous elementary acts of its implementation, known through experimental evidence.

To summarizing the above, we can say that:

- the birth of the Universe is not related to the appearance of an isolated cloud of matter consisting of elements which obey the laws of statistics;

- we do not know what vacuum is, but we do know that it played an important role in the birth of the Universe;

- elementary particles created in the act of the birth of the Universe should have a spatial distribution, adhering to central symmetry, and should have preferential velocity along the radial lines;

- act of the birth of the Universe could have lasted for a finite period of time within a finite volume of space with multiple, but finite number of simple spontaneous acts of "particle - antiparticle" pair generations;

- it is hypothetically possible that a set conditions exist that motivate elementary particles, appearing in simple spontaneous acts of "particle - antiparticle" pairs generations in the framework of the overall process of the birth of the Universe, to move only in the radial direction;

- the current level of the fundamental knowledge limits us to building schematic and hypothetical models of the birth of the Universe, without deciphering physical processes that have occurred at some point in the past, 
using trends rather than specific ways to implement them.

Currently not enough is known to even consider building a speculative abstract picture of the birth of the Universe. The main problem is the lack of a physical meaning of vacuum. Until an understanding of its nature can be reached, there is no point in creating models of the birth of the Universe. For example, under the characteristics of "uniform" and "isotropic" vacuum today we can understand only one thing: a uniform distribution of energy in space. Without the possibility of identifying the internal energy of the vacuum with any kind of energy, and without the possibility of understanding, even theoretically admissible methods of influence on the vacuum, the evidence remains completely abstract. To know how something can be affected, it is necessary to know what it is we are trying to affect.

\section{Possible Basis for a New Model of the Birth of the Universe}

If a uniform scalar field that fills the absolute void, for unknown reasons, would change in such a way that at a certain point of the vacuum as a result of the concentric perturbations the energy density would dramatically increase, then, in the absence of knowledge about the vacuum, we will be unable to guess what the nature of this disturbance might have been, and whether it could have been associated with a reduction in the energy density in the surrounding spherical layers of vacuum around the point of the increased energy density. Intuitively the answer would be yes. An increase in energy concentration may be accompanied by a symmetric loss of charge around it. However, this is only a guess based on general customary comparisons.

Let us assume that this situation did happen at some point in time in an infinite, homogeneous and isotropic vacuum. The reason for this assumption will soon become clear. Arisen energy gradients will induce a wave of spontaneous spherically symmetric acts of creation of "particle antiparticle" pairs, mainly - electron-positron pairs. One of the features of these acts will be not only a receding movement of electrons and positrons along radial lines, but matching directions of scattering for identical particles too. However, it is difficult to predict accurately, with current knowledge about the nature of vacuum, whether the electrons or positrons would be moving towards the center with high concentration of energy.

In any case, colliding with each other in the central area the particles will form a repeatedly electrically charged, completely unstable, excited composition, which must immediately disintegrate. The concentration of energy in the central zone will lead to the birth of "proton-antiproton" pairs and other sequential reactions, including annihilation, fulfilling of all mandatory conservation laws (energy, momentum, angular momentum, electric charge, baryon charge, lepton numbers), with the optional conservation laws (the strangeness and isospin) and take into account all available invariance principles (time reversal, spatial reflection and charge conjugation in different combinations).

The daughter particles of the central zone will have equally-probable directions of expansion. However, due to the small capacity in which daughter particles are generated, these arbitrary directions will coincide with the radial direction. It is acceptable to expect, on an intuitive level, that the acts of generation of electron-positron pairs will move in a spherical ripple (with an increase in diameter) with a declining number of new acts of generation of electron-positron pairs. New particles flying to the central zone of spontaneous generation will meet with the daughter particles that are moving away from the central zone, in head-on collisions. As a result an expanding cloud of matter will be formed, surrounded by scattering jets of electrons with relativistic speeds (if positrons collided in the central part of the initial disturbance of vacuum) or positrons (if electrons collided in the central zone, which seems most probable) and photons and antiphotons following them in radial directions, which will avoid head-on collisions with flying particles from the periphery. As shown in [4], a separated cloud of matter and a cloud surrounded by directional scattering matter are two fundamentally different starting situations to describe the subsequent gravitational interaction the elements of the cloud of matter with all matter in outer space (a consequence of Gauss' theorem on the absence of a gravitational field inside a closed cavity does not work in this case). A possible explanation of the accelerated divergence of matter in the Universe and the nature of the background electromagnetic radiation has appeared, for the discussed scheme of the birth of the Universe, in the framework of the classical gravitational theory without the involvement of physically indeterminable and not consciously recognizable objects and processes such as a singular point, dark energy, process of the expansion of time-space, abrupt simultaneous plasma recombination and so on.

\section{Conclusions}

In this work, after reviewing the current state of affairs, key assumptions are selected upon which a new cosmological model could be built and the principles are studied with which the new model of the birth of the Universe must comply. A possible framework (bearing structure) is provided as an example of the new model. However, no actual model is constructed. At the moment, there is not enough raw data and there is a lack of fundamental knowledge to build a harmonious model. A strict model can only be built in the future. It will then have to comply with all the requirements of the model, including its justification through a mathematical description.

It is clear that the above model can only be perceived as 
speculative. Something more profound is still fundamentally impossible to create. A natural question arises: "Why speculate, as this is not a scientific approach?" However, as has been shown above, that a model of the birth of the Universe does not exist to date: there is no model, not scientific, nor speculative. Therefore, even such an abstract way of constructing a model of the birth of the Universe is able to stimulate further search in terms of theoretical work. One should not only set goals, but also understand what real phenomena can be taken into consideration and what approximations are acceptable in the process of theoretical progress towards this goal. In other words, you need to understand what principles should be laid as foundations to create a model. Especially since now there is a new factor that needs to be taken into account - the existence of the halo around the Universe.

${ }^{\mathrm{i}}$ There is no information about affiliations because I am a retiree now. This is my own work made out of some kind of project.

\section{REFERENCES}

[1] D. S. Gorbunov, V. A. Rubakov. Introduction to the Theory of the Early Universe: Hot Big Bang Theory, World Scientific Publishing Company, 2011.

[2] D. S. Gorbunov, V. A. Rubakov. Introduction to the Theory of the Early Universe: Cosmological Perturbations and Inflationary Theory, World Scientific, Singapore, 2011.

[3] A. S. Belyaev. Photon gas, Universal Journal of Physics and Application, Horizon Research Publishing (HRPUB), Vol.10, No.1, 11-15, 2016. Online available from http://www.hrpub.o rg/download/20160229/UJPA3-18406008.pdf.

[4] A. S. Belyaev. Gravitational Contraction of a Dust Cloud in Space, Universal Journal of Physics and Application, Horizon Research Publishing (HRPUB), Vol.10, No.1, 22-30, 2016. Online available from http://www.hrpub.org/download/20160331/UJPA5-18406009 .pdf. 